\title{
Determining the risk factors associated with the development of Clostridium difficile infection in patients with hematological diseases
}

\author{
Yu Ling Lee-Tsai ${ }^{1}$, Rodrigo Luna-Santiago ${ }^{1}$, Roberta Demichelis-Gómez ${ }^{1}$, Alfredo Ponce-de-León ${ }^{2}$, \\ Eric Ochoa-Hein ${ }^{3}$, Karla María Tamez-Torres ${ }^{2}$, María T Bourlon ${ }^{1}$, Christianne Bourlon ${ }^{1}$ \\ ${ }^{1}$ Department of Hematology and Oncology, ${ }^{2}$ Department of Infectology, ${ }^{3}$ Departament of Hospital Epidemiology and Quality Control \\ of Medical Care, Instituto Nacional de Ciencias Médicas y Nutrición Salvador Zubirán, Mexico City, Mexico
}

p-ISSN 2287-979X / e-ISSN 2288-0011 https://doi.org/10.5045/br.2019.54.2.120 Blood Res 2019;54:120-124.

Received on December 14, 2018

Revised on February 26, 2019

Accepted on February 28, 2019
*This study was supported by a grant from Aramont A.C. Foundation.

\section{Correspondence to}

Christianne Bourlon, M.D.

Department of Hematology and Oncology, Instituto Nacional de Ciencias Médicas y Nutrición Salvador Zubirán, Vasco de Quiroga No. 15, Belisario Domínguez Sección XVI, Tlalpan, C.P. 14080, Mexico City, Mexico

E-mail: chrisbourlon@hotmail.com

(C) 2019 Korean Society of Hematology

\section{Background}

Clostridium difficile infection (CDI) is a nosocomial condition prevalent in patients with hematological disorders. We aimed to identify the risk factors associated with the development of $\mathrm{CDI}$ and assess the mortality rate at 15 and 30 days among hematologic patients admitted to a tertiary care center.

Methods

We conducted a retrospective case-control study from January 2010 to December 2015. Forty-two patients with hematologic malignancy and CDI, and 84 with hematologic disease and without history of CDI were included in the case and control groups, respectively.

Results

Univariate analysis revealed that episodes of febrile neutropenia [odds ratio (OR), 5.5; 95\% confidence interval $(\mathrm{Cl}), 2.3-12.9 ; P<0.001$ ], admission to intensive care unit (OR, $3.8 ; 95 \% \mathrm{Cl}, 1.4-10.2 ; P=0.009)$, gastrointestinal surgery $(\mathrm{OR}, 1.2 ; 95 \% \mathrm{Cl}, 1.1-1.4$; $P<0.001)$, use of therapeutic $(\mathrm{OR}, 6.4 ; 95 \% \mathrm{Cl}, 2.5-15.9 ; P<0.001)$ and prophylactic antibiotics (OR, $4.2 ; 95 \% \mathrm{Cl}, 1.6-10.7 ; P=0.003)$ in the last 3 months, and $>1$ hospitalization (OR, 5.6; 95\% Cl, 2.5-12.6; $P<0.001$ ) were significant risk factors. Multivariate analysis showed that use of therapeutic antibiotics in the last 3 months (OR, 6.3; 95\% $\mathrm{Cl}, 2.1-18.8 ; P=0.001)$ and $>1$ hospitalization $(\mathrm{OR}, 4.3 ; 95 \% \mathrm{Cl}, 1.7-11.0 ; P=0.002)$ were independent risk factors. Three (7.1\%) and $6(14.2 \%)$ case patients died at 15 and 30 days, respectively.

\section{Conclusion}

The risk factors for developing CDI were exposure to therapeutic antibiotics and previous hospitalization. Hematological patients who developed CDI had higher early mortality rates, suggesting that new approaches for prevention and treatment are needed.

Key Words Clostridium difficile, Hematologic diseases, Risk factors, Tertiary care centers

\section{INTRODUCTION}

Clostridium difficile (CD) is an anaerobic, Gram-positive, spore-forming bacillus capable of colonizing and proliferating in the human intestinal epithelium [1]. CD strains that produce toxins $\mathrm{A}$ and $\mathrm{B}$ appear to play a major role in the occurrence of clinical manifestations. CD infection (CDI) may manifest in a wide range of symptoms, from an asympto- matic carrier and mild diarrhea to the development of pseudomembranous colitis [1-4]. In western countries, an incidence of 2-6 cases per 10,000 hospitalized patients per day has been reported, with CD regarded as the main cause of nosocomial infections [1-3]. In the Latin American population, a multicenter study reported a rate of 11 per 10,000 hospitalized patients over 40 years of age per day [5]; in Mexico, rates of $2.8 \mathrm{CD}$ cases per 10,000 hospitalized patients a day and 1.5 cases per 1,000 admissions have been reported 
[6].

Antibiotic use is considered as the risk factor that is most likely associated with CDI, particularly the use of clindamycin and fluoroquinolones [1-4]. Other variables that have been described as risk factors in the general population include previous hospitalizations [3,7], age $>65$ years [7], inflammatory bowel disease [8], nasogastric tube feeding [9], GI surgery [9], obesity $[8,9]$, antineoplastic therapy $[8,9]$, hematopoietic stem cells transplantation (HSCT) $[8,9]$, use of gastric-acid suppressing drugs [8,9], and admission to the ICU [9]. At the microbiological level, infection caused specifically by the NAP1/BI/027 CD ribotype strain has been associated with a higher incidence of severity and recurrence in some populations $[1,8-10]$.

Patients with hematological diseases are at higher risk of developing CDI, partly because they undergo intensive chemotherapy (CT) and/or bone marrow failure that can lead to immunosuppression [11]. In addition, many patients require prolonged hospitalizations and are exposed to multiple drugs, including broad-spectrum antibiotics $[11,12]$. Different research groups have reported higher incidence rates of CDI among hematological patients in comparison to other patient groups [13-19].

CDI has become a major concern for physicians and health systems due to its increasing morbidity and mortality. In Latin America, little is known about the risk factors associated with the development of CDI in patients with hematological disorders. Therefore, the study aimed to identify the risk factors associated with its development and assess the mortality rate at 15 and 30 days among Latin American hematologic patients admitted in a tertiary care center.

\section{MATERIALS AND METHODS}

\section{Patients}

The study participants were patients aged $\geq 15$ years diagnosed with hematological malignancy (acute leukemia, multiple myeloma, or lymphoma) or bone marrow failure (aplastic anemia or myelodysplastic syndrome) according to the World Health Organization (WHO) 2016 criteria [20]. The study was approved by the local Research Ethics Board.

Patients with CDI who were attended at our institution from January 2010 to December 2015 were included in this study. For each case, two controls were assigned, matched by hematological diagnosis, same year of diagnosis, and date of birth within the same year. The intensity of the therapeutic regimens was defined according to the Common Terminology Criteria for Adverse Events (CTCAE, version 5.0) [21] for myelotoxicity: high intensity (grade 4), intermediate intensity (grades 2 and 3), and low intensity or no CT (grade 1).

CDI was defined as the acute onset of diarrhea (defecation $\geq 3$ times per day or at least $200 \mathrm{~g}$ of stool per day) [22] plus a positive fecal sample for $\mathrm{CD}$ toxin $\mathrm{A}$ and/or $\mathrm{B}$ by enzyme immunoassay [23] or tissue culture neutralization assay and/or a positive stool sample for a CD producer of toxins detected by PCR or culture [24, 25]. CD ribotype was determined by a PCR-based assay (Cepheid Xpert $C$. difficile/EpI). The severity of each episode of diarrhea was classified as mild-moderate, severe, and complicated infection according to the 2010 criteria of the Infectious Disease Society of America (IDSA) for the management of CDI [10]. Initial treatment for $\mathrm{CDI}$ was based on the attending physician's choice. Response to treatment was defined as resolution of diarrhea 10-14 days after treatment initiation [22]. Deterioration of abdominal symptoms, persistence of diarrhea after 14 days of treatment, need for additional antibiotic therapy, and progression to severe enterocolitis were considered as treatment failure [10]. Patients who developed diarrhea 2 weeks after resolution of the previous clinical episode were classified as having relapse-recurrence $[10,16,18,26]$. Mortality was recorded at 15 and 30 days, and cause of death was determined using the death certificate.

\section{Statistical analysis}

The categorical variables were expressed as frequencies and proportions, while the continuous variables were expressed as medians and ranges. Logistic regression analysis was performed to identify the risk factors. The SPSS version

Table 1. Patients' baseline characteristics.

\begin{tabular}{|c|c|c|}
\hline Characteristics & $\begin{array}{l}\text { Cases } \\
(\mathrm{N}=42)\end{array}$ & $\begin{array}{l}\text { Controls } \\
(\mathrm{N}=84)\end{array}$ \\
\hline Median age, yr (range) & $51(17-90)$ & $51(15-86)$ \\
\hline \multicolumn{3}{|l|}{ Sex, N (\%) } \\
\hline Male & $20(47.6)$ & $41(48.8)$ \\
\hline Female & $22(52.4)$ & $43(51.2)$ \\
\hline \multicolumn{3}{|l|}{ Hematological diagnoses, N (\%) } \\
\hline Acute leukemia & $22(52.4)$ & $44(52.4)$ \\
\hline Lymphoma/multiple myeloma & $15(35.7)$ & $30(35.7)$ \\
\hline Bone marrow failure & $5(11.9)$ & 10 (11.9) \\
\hline \multicolumn{3}{|l|}{ Chemotherapy intensity, N (\%) } \\
\hline High & $22(52.4)$ & $40(47.6)$ \\
\hline Intermediate & $9(21.4)$ & $28(33.3)$ \\
\hline Low/no chemotherapy & $11(26.2)$ & $16(19.0)$ \\
\hline Use of steroids, N (\%) & $26(61.9)$ & $55(65.5)$ \\
\hline Previous hospitalizations, N (range) & $2.5(1-7)$ & $1(0-5)$ \\
\hline Gastrointestinal surgery, N (\%) & $8(19)$ & $0(0)$ \\
\hline Admission to the ICU, $\mathrm{N}(\%)$ & $12(28.6)$ & $8(9.5)$ \\
\hline Febrile neutropenia, $\mathrm{N}(\%)$ & $20(47.6)$ & $12(14.3)$ \\
\hline Used antibiotics within $3 \mathrm{mo}, \mathrm{N}(\%)$ & $35(83.3)$ & $37(44)$ \\
\hline $\begin{array}{l}\mathrm{N} \text { of antibiotics used over the last } \\
3 \mathrm{mo} \text {, median (range) }\end{array}$ & $3(0-5)$ & $0(0-5)$ \\
\hline Penicillin, N (\%) & $22(52.4)$ & $24(28.6)$ \\
\hline Quinolone, N (\%) & $7(16.7)$ & $9(10.7)$ \\
\hline Cephalosporin, N (\%) & $10(23.8)$ & $5(6)$ \\
\hline Clindamycin, N (\%) & $3(7.1)$ & $1(1.2)$ \\
\hline Macrolide, N (\%) & $4(9.5)$ & $4(4.8)$ \\
\hline Metronidazole, N (\%) & $7(16.7)$ & $8(9.5)$ \\
\hline Vancomycin, N (\%) & $21(50)$ & $26(31)$ \\
\hline Carbapenem, N (\%) & $23(54.8)$ & $26(31)$ \\
\hline
\end{tabular}

Abbreviation: ICU, intensive care unit. 
22 software (IBM Corp, Armonk, NY) was used, and statistical significance was defined as $P \leq 0.05$.

\section{RESULTS}

About 42 cases and 84 controls were included in the study. The median age of the patients was 51 years (15-90), and $51.6 \%(\mathrm{~N}=65)$ were women. About 66 patients $(52.4 \%)$ were diagnosed with acute leukemia, 45 (35.7\%) with lymphoma or multiple myeloma (MM), and 15 (11.9\%) with bone marrow failure. With regard to the therapeutic regimens used for the primary diagnosis, $49.2 \%$ of the patients received $(\mathrm{N}=62)$ high intensity $\mathrm{CT}, 29.4 \%(\mathrm{~N}=37)$ received intermediate intensity $\mathrm{CT}$, and $21.4 \%(\mathrm{~N}=27)$ received low intensity or no CT. Additional baseline characteristics are presented in Table 1.

Approximately 50\% ( $\mathrm{N}=21)$ of CDI episodes were classified as having severe-complicated infections, with $23.8 \%(\mathrm{~N}=10)$ associated with hemodynamic instability. The first-line antibiotic treatment was oral (PO) metronidazole in $29 \%(\mathrm{~N}=12)$, intravenous (IV) metronidazole in $29 \%(\mathrm{~N}=12)$, PO vancomycin in $17 \%(\mathrm{~N}=7)$, and IV metronidazole plus $\mathrm{PO}$ vancomycin in $26 \%(\mathrm{~N}=11)$. Overall, $9.5 \%(\mathrm{~N}=4)$ of the patients required surgical intervention due to toxic megacolon. $\mathrm{CD}$ ribotype 027 was present in $16.7 \%(\mathrm{~N}=7)$ of patients but was not associated with worse clinical outcomes.

A total of 35 patients (83.3\%) responded well to the initial treatment, with $28.6 \%(\mathrm{~N}=12)$ presenting relapse during the follow-up period. Mortality rates of all groups at 15 and 30 days were $4.8 \%(\mathrm{~N}=6)$ and $10.3 \%(\mathrm{~N}=13)$, respectively. Mortality rates of the CDI group at 15 and 30 days were $7.1 \%(\mathrm{~N}=3)$ and $14.3 \%(\mathrm{~N}=6)$, whereas those of the control group were $3.6 \%(\mathrm{~N}=3)$ and $8.3 \%(\mathrm{~N}=7)$, respectively (Fig. $1)$. As regards the causes of death among the case group, $50 \%$ of deaths $(\mathrm{N}=3)$ were due to the CDI episode, $33.3 \%$ $(\mathrm{N}=2)$ were due to nosocomial pneumonia, and $16.6 \%(\mathrm{~N}=1)$ were due to bacteremia.

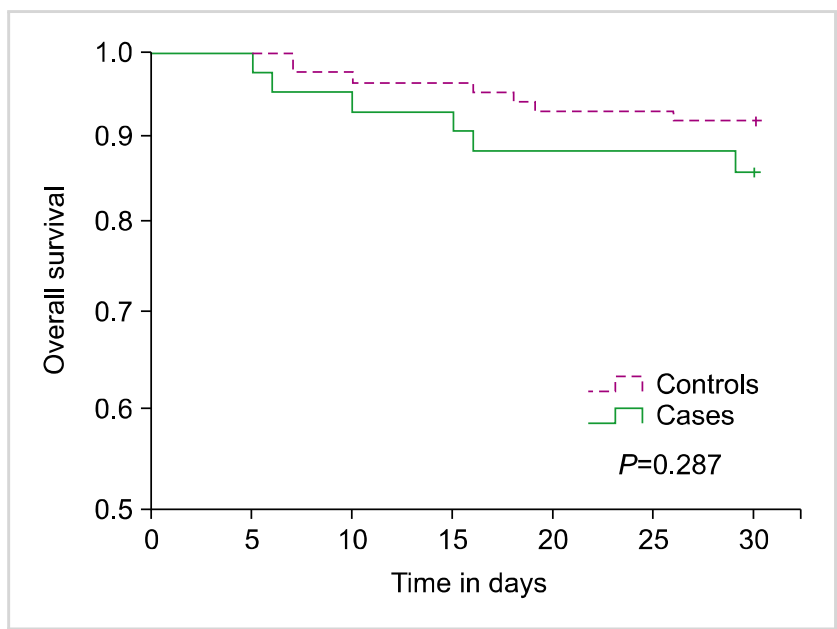

Fig. 1. Overall survival of cases and controls at 15 and 30 days.
In the univariate analysis, significant risk factors for the development of CDI were previous episodes of febrile neutropenia (OR, 5.5; 95\% CI, 2.3-12.9; $P<0.001$ ), previous ICU admission (OR, 3.8; 95\% CI, 1.4-10.2; $P=0.009$ ), previous GI surgery (OR, 1.2; 95\% CI, 1.1-1.4; $P<0.001$ ), use of therapeutic antibiotics (OR, 6.4; 95\% CI, 2.5-15.9; $P<0.001)$ and prophylactic antibiotics (ciprofloxacin and trimethoprim/sulfamethoxazole) (OR, 4.2; 95\% CI, 1.6-10.7; $P=0.003$ ) in the last 3 months, and more than one previous hospitalization (OR, 5.6; 95\% CI, 2.5-12.6; $P<0.001$ ). Among the antibiotics used, those with significant statistical association were penicillin (OR, 2.8; 95\% CI, 1.3-5.9; $P=0.011$ ), cephalosporin (OR, 4.9; 95\% CI, 1.6-15.6; $P=0.007$ ), vancomycin (OR, 2.2; 95\% CI, 1.0-4.8; $P=0.05$ ), and carbapenem (OR, 2.7; 95\% CI, 1.3-5.8; $P=0.011$ ) (Table 2). In the multivariate analysis, independent risk factors were the use of therapeutic antibiotics in the previous 3 months (OR, 6.3; 95\% CI, 2.118.8; $P=0.001)$ and more than one previous hospitalization (OR, 4.3; 95\% CI, 1.7-11.0; $P=0.002$ ).

\section{DISCUSSION}

In this case-control study, we found that the risk factors related to the development of CDI in our patients with hematological diseases did not differ from those reported in the studies conducted in other populations $[1-4,7]$. Considering the existing knowledge on the pathogenesis of CDI, which involves exogenous acquisition from the transmission of bacterial spores, the associations identified among our study

Table 2. Univariate analysis of the risk factors associated with the development of $\mathrm{CDI}$ in patients with hematological diseases.

\begin{tabular}{llr}
\hline \multicolumn{1}{c}{ Characteristics } & \multicolumn{1}{c}{$\begin{array}{c}\text { Univariate } \\
\text { Prob }(95 \% \mathrm{Cl})\end{array}$} & \multicolumn{1}{c}{$P$} \\
\hline Febrile neutropenia & $5.5(2.3-12.9)$ & $<0.001$ \\
Previous anti-cancer chemotherapy & $0.8(0.4-1.8)$ & 0.687 \\
Steroids & $0.9(0.4-1.8)$ & 0.844 \\
Admission to ICU & $3.8(1.4-10.2)$ & 0.009 \\
Gl surgery & $1.2(1.1-1.4)$ & $<0.001$ \\
Tx antibiotics & $6.4(2.5-15.9)$ & $<0.001$ \\
Pr antibiotics & $4.2(1.6-10.7)$ & 0.003 \\
Penicillin & $2.8(1.3-5.9)$ & 0.011 \\
Quinolone & $1.7(0.6-4.8)$ & 0.399 \\
Cephalosporin & $4.9(1.6-15.6)$ & 0.007 \\
Clindamycin & $6.4(0.6-63.3)$ & 0.108 \\
Macrolide & $2.1(0.5-8.9)$ & 0.439 \\
Sulfonamide & $1.0(0.9-1.1)$ & 0.333 \\
Metronidazole & $1.9(0.6-5.7)$ & 0.189 \\
Vancomycin & $2.2(1.0-4.8)$ & 0.050 \\
Carbapenem & $2.7(1.3-5.8)$ & 0.011 \\
Hospitalizations $>1$ & $5.6(2.5-12.6)$ & $<0.001$ \\
PPI & $2.0(0.9-4.4)$ & 0.087 \\
Prokinetics & $0.3(0.1-1.0)$ & 0.073
\end{tabular}

Abbreviations: $\mathrm{Gl}$, gastrointestinal; ICU, intensive care unit; PPI, proton pump inhibitors; $\mathrm{Pr}$, prophylactic; $\mathrm{Tx}$, therapeutic. 
variables can be substantiated.

Previous hospitalization is a risk factor for the acquisition of CDI, and the probability of developing this pathology is directly proportional to the length of hospital stay, with reported acquisition rates of up to $50 \%$ in patients hospitalized for more than 4 weeks [1, 27]. Additionally, CDI is an indicator of medical care quality as its development can double hospitalization time and/or the number of hospital admissions per patient in the population affected by hematologic neoplasms [15, 28].

Previous use of any type of antibiotic is one of the main factors associated with the development of CDI in hematological patients $[9,29]$. As the patients are exposed to intensive CT and many infectious processes, they exhibit a greater susceptibility to CDI development $[13,14,16,18$, 28]. Notably, no previous study carried out in the hematological population has separately analyzed the association between prophylactic and therapeutic antibiotic use and development of CDI. In our study, the use of therapeutic antibiotics and prophylactic antibiotics in the last 3 months had statistically significant associations with CDI in the univariate analysis, and therapeutic antibiotic use remained statistically significant in the multivariate analysis. Unlike previously reported data, antimicrobials including penicillin, cephalosporin, vancomycin, and carbapenem were shown to be independent risk factors based on the statistically significant associations with CDI in the univariate analysis. On the contrary, Gweon et al. [14] found that the total number of antibiotics used and their duration of use were greater in patients with hematological diseases who developed CDI compared with non-hematological patients, adding greater external validity to the previously proposed hypothesis. Similarly, decrease exposure to antimicrobial agents was associated with a higher rate of CDI resolution [14].

Fuereder et al. [29] reported their results on the risk for CDI in a cohort of patients with hematological and oncologic malignancies. Consistent with our results, they found that the use of antimicrobial agents within the previous month was an independent risk factor, whereas the use of CT was not related to the development of CDI. However, the population included was widely heterogeneous in terms of chemotherapeutic regimens used, with different myelotoxicity and gastrointestinal toxicity potential.

Compared with the results of previous studies, half of the CDI cases in our study experienced severe-complicated episodes and higher rates of complications $[14,16]$. Interestingly, in our study group, the mortality rate attributed to CDI was similar to that reported in the literature $(2.4 \%)$, being the higher mortality rate attributed to other concomitant infectious complications $[14,16,18,26,30]$. Our explanation to this finding is based on the hypothesis that our study population has a higher risk of developing infectious complications because of the advance stage of disease at diagnosis, and that the first-line treatment for CDI episodes was PO metronidazole (according to the 2010 recommendations of the IDSA) [10]. However, the current recommendation for first-line treatment is $\mathrm{PO}$ vancomycin or fi- daxomicin, and PO metronidazole is used as treatment for mild-moderate episodes [31].

The prevalence of mortality at 30 days ranged between $16 \%$ and $33 \%$ in other studies, while that in our study was $14.3 \%[14,19,24,29]$. In those studies, the specific cause of death in one patient was attributed only to the CDI episode $(0.7 \%)$, and the rest of the patients died with an active CDI caused by other factors $[14,26]$. Among the reported deaths related to the CDI episode $(\mathrm{N}=3)$, only one case was directly attributed to this event (2.4\%); the other patients had bacteremia due to infection with Klebsiella pneumoniae and Candida albicans, and the last patient had soft tissue infection, Escherichia coli bacteremia, and nosocomial pneumonia (4.8\%).

Although the main limitation of this study is its retrospective nature, it provides relevant evidence suggesting that hematological patients are highly susceptible to not only CDI but also to the development of complications arising from this infection and/or concomitant complications that can directly impact early mortality rates $[13,14,16]$. Therefore, a higher proportion of recurrence and mortality was difficult to assess over a longer period of time. Although the mortality rate was higher in patients with CDI than in controls, we did not find a statistically significant association because of the sample size.

The main strength of this study is that we were able to explore new variables that have not been previously studied, such as febrile neutropenia. Additionally, we separately analyzed those patients treated with prophylactic antibiotics from those receiving therapeutic antibiotics; both factors are important in the context of a hematologic patient receiving CT with an increased risk of infections and with a statistically significant association with CDI.

New prospective studies that will analyze the variables found and with longer follow-ups in hematologic patients who developed CDI are necessary to demonstrate a causal association between risk factors and CDI and can propose new approaches for prevention and treatment.

\section{ACKNOWLEDGMENTS}

We acknowledge the Aramont A. C. Foundation for the support of this research and Dr. Lilian Sung from the Division of Hematology and Oncology, the Hospital for Sick Children, University of Toronto, Ontario, Canada.

\section{Authors' Disclosures of Potential Conflicts of Interest}

No potential conflicts of interest relevant to this article were reported.

\section{REFERENCES}

1. Martin JS, Monaghan TM, Wilcox MH. Clostridium difficile 
infection: epidemiology, diagnosis and understanding transmission. Nat Rev Gastroenterol Hepatol 2016;13:206-16.

2. Surawicz CM, Brandt LJ, Binion DG, et al. Guidelines for diagnosis, treatment, and prevention of Clostridium difficile infections. Am J Gastroenterol 2013;108:478-98.

3. Khan FY, Elzouki AN. Clostridium difficile infection: a review of the literature. Asian Pac J Trop Med 2014;7S1:S6-13.

4. Kachrimanidou M, Malisiovas N. Clostridium difficile infection: a comprehensive review. Crit Rev Microbiol 2011;37:178-87.

5. Lopardo G, Morfin-Otero R, Moran-Vazquez II, et al. Epidemiology of Clostridium difficile: a hospital-based descriptive study in Argentina and Mexico. Braz J Infect Dis 2015;19:8-14.

6. Dávila LP, Garza-González E, Rodríguez-Zulueta P, et al. Increasing rates of Clostridium difficile infection in Mexican hospitals. Braz J Infect Dis 2017;21:530-4.

7. Dupont HL. Diagnosis and management of Clostridium difficile infection. Clin Gastroenterol Hepatol 2013;11:1216-23.

8. Dubberke ER, Reske KA, Yan Y, Olsen MA, McDonald LC, Fraser VJ. Clostridium difficile--associated disease in a setting of endemicity: identification of novel risk factors. Clin Infect Dis 2007;45:1543-9.

9. Camacho-Ortiz A, Galindo-Fraga A, Rancel-Cordero A, et al. Factors associated with Clostridium difficile disease in a tertiarycare medical institution in Mexico: a case-control study. Rev Invest Clin 2009;61:371-7.

10. Cohen SH, Gerding DN, Johnson S, et al. Clinical practice guidelines for Clostridium difficile infection in adults: 2010 update by the Society for Healthcare Epidemiology of America (SHEA) and the Infectious Diseases Society of America (IDSA). Infect Control Hosp Epidemiol 2010;31:431-55.

11. Anand A, Glatt AE. Clostridium difficile infection associated with antineoplastic chemotherapy: a review. Clin Infect Dis 1993;17:109-13.

12. Peretz A, Shlomo IB, Nitzan O, Bonavina L, Schaffer PM, Schaffer M. Clostridium difficile infection: associations with chemotherapy, radiation therapy, and targeting therapy treatments. Curr Med Chem 2016;23:4442-9.

13. Gu SL, Chen YB, Lv T, et al. Risk factors, outcomes and epidemiology associated with Clostridium difficile infection in patients with haematological malignancies in a tertiary care hospital in China. J Med Microbiol 2015;64:209-16.

14. Gweon TG, Choi MG, Baeg MK, et al. Hematologic diseases: high risk of Clostridium difficile associated diarrhea. World J Gastroenterol 2014;20:6602-7.

15. Selvey LA, Slimings C, Joske DJ, Riley TV. Clostridium difficile infections amongst patients with haematological malignancies: A data linkage study. PLoS One 2016;11:e0157839.

16. Parmar SR, Bhatt V, Yang J, Zhang Q, Schuster M. A retrospective review of metronidazole and vancomycin in the management of Clostridium difficile infection in patients with hematologic malignancies. J Oncol Pharm Pract 2014;20:172-82.

17. Altclas J, Requejo A, Jaimovich G, Milovic V, Feldman L. Clostridium difficile infection in patients with neutropenia. Clin Infect Dis 2002;34:723.

18. Schalk E, Bohr UR, König B, Scheinpflug K, Mohren M.
Clostridium difficile-associated diarrhoea, a frequent complication in patients with acute myeloid leukaemia. Ann Hematol 2010; 89:9-14.

19. Spadão F, Gerhardt J, Guimarães T, et al. Incidence of diarrhea by Clostridium difficile in hematologic patients and hematopoietic stem cell transplantation patients: risk factors for severe forms and death. Rev Inst Med Trop Sao Paulo 2014;56:325-31.

20. Swerdlow SH, Campo E, Harris NL, et al, eds. WHO classification of tumours of haematopoietic and lymphoid tissues. Revised 4th ed. Lyon, France: IARC Press, 2017.

21. National Institutes of Health. Common Terminology Criteria for Adverse Events (CTCAE) Version 5.0. Bethesda, MD: National Institutes of Health, 2017. (Accessed September 18, 2018, at https://ctep.cancer.gov/protocolDevelopment/electronic_appli cations/docs/CTCAE_v5_Quick_Reference_8.5x11.pdf

22. Shane AL, Mody RK, Crump JA, et al. 2017 infectious diseases society of america clinical practice guidelines for the diagnosis and management of infectious diarrhea. Clin Infect Dis 2017;65: e45-80.

23. Eckert C, Said O, Rambaud C, et al. Comparison of the VIDAS ${ }^{\circledR}$ C. difficile GDH and the GDH component of the C. diff Quik Chek Complete for detection of Clostridium difficile in stools. ECCMID (Annual Congress Abstracts) 2013;eP1871.

24. CM0601. Clostridium difficile agar base. Basingstoke, UK: Thermo Fisher Scientific Inc, 2013. (Accessed September 18, 2018, at http://www.oxoid.com/UK/blue/prod_detail/prod_detail. asp?pr=CM0601\&org=52\&c=UK\&lang=EN).

25. Xpert ${ }^{\mathbb{R}}$ C. difficile/Epi. 45-minute detection \& differentiation of clostridium difficile \& the Epidemic 027 strain. Sunnyvale, CA: Cepheid, 2018. (Accessed September 18, 2018, at http:// www.cepheid.com/us/cepheid-solutions/clinical-ivd-tests/heal thcare-associated-infections/xpert-c-difficile-epi).

26. Gorschlüter M, Glasmacher A, Hahn C, et al. Clostridium difficile infection in patients with neutropenia. Clin Infect Dis 2001; 33:786-91.

27. Clabots CR, Johnson S, Olson MM, Peterson LR, Gerding DN. Acquisition of Clostridium difficile by hospitalized patients: evidence for colonized new admissions as a source of infection. J Infect Dis 1992;166:561-7.

28. Apostolopoulou E, Raftopoulos V, Terzis K, Elefsiniotis I. Infection Probability Score: a predictor of Clostridium difficile-associated disease onset in patients with haematological malignancy. Eur J Oncol Nurs 2011;15:404-9.

29. Fuereder T, Koni D, Gleiss A, et al. Risk factors for Clostridium difficile infection in hemato-oncological patients: A case control study in 144 patients. Sci Rep 2016;6:31498.

30. Yoon YK, Kim MJ, Sohn JW, et al. Predictors of mortality attributable to Clostridium difficile infection in patients with underlying malignancy. Support Care Cancer 2014;22:2039-48.

31. McDonald LC, Gerding DN, Johnson S, et al. Clinical Practice Guidelines for Clostridium difficile Infection in Adults and Children: 2017 Update by the Infectious Diseases Society of America (IDSA) and Society for Healthcare Epidemiology of America (SHEA). Clin Infect Dis 2018;66:e1-48. 